\title{
The urethra is a reliable witness: simplifying the diagnosis of stress urinary incontinence. Reply to Wagenlehner et al.
}

\author{
Charles Nager
}

Published online: 23 March 2013

(C) The International Urogynecological Association 2013

Dear Editor,

Dr. Wagenlehner and colleagues commented on my editorial, "The urethra is a reliable witness: simplifying the diagnosis of stress urinary incontinence" [1] by proposing a unilateral midurethral anchor test to predict the outcome after midurethral sling surgery. They present ultrasound observations that this procedure can prevent "funneling" of the bladder neck and urine loss, and they present urodynamic urethral pressure profilometry observations that this procedure increases maximum urethral closure pressure and improves the cough pressure transmission ratio. Similar urodynamic observations were reported 30 years ago by Bhatia and Bergman with the Bonney test [2] and the Marshall-Marchetti test [3], and I suspect the observations by Wagenlehner and colleagues are correct. Whether this test accurately reproduces what happens during a midurethral sling surgery or how predictive it is for surgical outcomes would require studies involving a standardized preoperative performance of this test in a large number of patients who have a midurethral sling so that positive and negative predictive values for the midurethral anchor test could be calculated.

I do disagree with the statement that Wagenlehner et al. made in their reply with regards to the standing cough stress test. They stated: "We agree, but in our experience, testing in the standing position confers no additional accuracy against

This reply refers to the comment available at doi 10.1007/s00192-0132059-1.

\section{Nager $(\bowtie)$}

UC San Diego Health System, 9350 Campus Point Dr, Ste 2A,

Mail Code 0974,

La Jolla, CA 92037-1300, USA

e-mail: cnager@mail.ucsd.edu testing in the supine position." The experience of myself and many others is quite different. The symptom of stress urinary incontinence (SUI) in women is more likely to occur in the upright than the supine position. Bladder pressure is higher when standing than when supine. In the Outcomes following vaginal prolapse repair and mid urethral sling (OPUS) trial of women with prolapse but without symptoms of incontinence, 111 of 331 women had a positive preoperative reduction stress test, but in 33 , this only became positive in the standing position [4]. When a positive stress test includes both supine and standing positive results, the test predicts a $72 \%$ rate of postoperative SUI at 3 months and a $60 \%$ rate at 12 months if a midurethral sling is not inserted at the time of vaginal prolapse surgery (Supplementary Table 5) [5]. To maximize the sensitivity of the stress test in detecting SUI, it should be done standing with a full bladder.

\section{References}

1. Nager CW (2012) The urethra is a reliable witness: simplifying the diagnosis of stress urinary incontinence. Int Urogynecol J 23 (12):1649-51. doi:10.1007/S00192-012-1892-y

2. Bhatia NN, Bergman A (1983) Urodynamic appraisal of the Bonney test in women with stress urinary incontinence. Obstet Gynecol 62 (6):696-9

3. Bergman A, Bhatia NN (1987) Urodynamic appraisal of the Marshall-Marchetti test in women with stress urinary incontinence. Urology 29(4):458-62

4. Nager C (2011) The Value of the Preoperative Prolapse Reduction Stress Test in Women without Stress Incontinence Symptoms Undergoing Vaginal Prolapse Surgery with or without a TVT: Results from the OPUS Trial. Female Pelvic Medicine and Reconstructive Surgery 17(Number 5, Supplement 2):53

5. Wei JT et al (2012) A midurethral sling to reduce incontinence after vaginal prolapse repair. N Engl J Med 366(25):2358-67 